\title{
Cardioprotective properties of fish enriched with bioactive lipids from olive oil by products
}

\section{Abstract}

Anti-inflammatory properties of olive oil are partly attributed to the inhibition of the Platelet-Activating Factor (PAF), a potent inflammatory mediator that is implicated in the initiation and prolongation of atherosclerosis. PAF inhibitors also exist in an extract from olive pomace that inhibits development and regression of atherosclerotic plaques in hypercholesterolemic rabbits. On the other hand, fish consumption is recommended for prevention of cardiovascular diseases, leading to a constantly increasing demand for fish supply, which is further enhanced by the rising consumers' awareness. As a result a significant growth of aquaculture worldwide has been occurred, which among others strives for improving the nutritional value of the produced fish. Important breakthroughs have occurred in the replacement of fish oil, traditionally used, by plant oils in compounded fish feeds contributing to more nutritious fish products. Under this perspective, present work aimed to evaluate the cardioprotective properties of a novel seabream fed with olive pomace extract.

In this crossover study, thirty apparently healthy, men and women were randomly assigned in two diet sequences for 8-week duration (6-week washout period between them), consuming either normal or enriched with olive pomace fish, twice a week. The study was approved by the Bioethics Committee of Harokopio University. Dietary intake was assessed with a validated for the Greek population semi-quantitative food-frequency questionnaire. Lifestyle parameters, biochemical factors, P-selectin and Plasminogen-activator inhibitor-1 (PAI-1) levels were determined. In addition, ex-vivo platelet aggregation in platelet-rich plasma against PAF, ADP and thrombin were measured and the results expressed as $\mathrm{EC}_{50}$ values (the concentration of the agonist that cause $50 \%$ of maximum aggregation). Also, PAF key metabolic enzymes activities, responsible for PAF levels, were determined.

Concerning dietary habits and biochemical markers only total cholesterol revealed a small increase, within the normal range, after enriched fish consumption, compared to baseline levels. Both fishes resulted in higher $\mathrm{EC}_{50}$ (lower platelet sensitivity) values only against PAF without being differentiated $\left(\mathrm{p}_{\text {time }}=0.02, \mathrm{p}_{\text {trial }}=0.9\right)$, while PAI-1 levels were only increased after normal fish consumption $\left(\mathrm{p}_{0-8}\right.$ weeks $\left.=0.01\right)$. The ratio of Lipoprotein-Associated-Phospholipase $\mathrm{A}_{2}$ (catabolic enzyme of PAF) to LDL and PAF-CDP-choline:cholinephosphotransferase (biosynthetic enzyme of PAF) were decreased after the enriched $\left(\mathrm{p}_{0-8}\right.$ weeks $\left.=0.003\right)$ and the normal $\left(\mathrm{p}_{0-8}\right.$ weeks $\left.=0.01\right)$ fish consumption, respectively.

Seabream fed with olive pomace seems to exhibit cardioprotective properties by decreasing platelet sensitivity against PAF and modulate PAF metabolism.

This research has been co-financed by the European Regional Development Fund of the EU and Greek national funds (project code:T1EDK-00687).

\section{Conflict of Interest}

There is no conflict of interest 